\title{
A Study on the Basic Situation of PE Teachers in the Background of Sports senior high school entrance examination reform in urban middle school Jilin province
}

\author{
Guo $\mathrm{Lu}^{1, \text { a }}$, Xianhong $\mathrm{Lin}^{2, \text { a }}$ and Dan $\mathrm{Li}^{3, \mathrm{a}}$ \\ ${ }^{1}$ Jilin Agricultural University, Xincheng Street 2888, Changchun, Jilin Province 130118, PR China \\ 2,3 Jilin Sport University, 2476 Ziyou Road, Changchun City, Jilin Province 130118, PR China \\ a360282476@qq.com
}

Keywords: Sports senior high school entrance examination; Reform; Physical education teachers; Team building

\begin{abstract}
With the graduation of sports subjects in the examination will gradually increase the score, Sports senior high school entrance examination reform and continuous improvement, will focus on the development of sports quality period. This paper analyzes the physical education teachers of urban middle schools in Jilin Province, explores the situation of PE teachers in the background of the reform of the examination, and improves the comprehensive knowledge structure, so as to promote the comprehensive quality of the secondary schools in Jilin Province.

National provinces and cities in the examination are equipped with sports senior high school entrance examination projects, and now the total score in the development trend is the sports score in the slowly upgrade.
\end{abstract}

\section{Basic Situation of PE Teachers in the Background of Sports Senior High School Entrance Examination Reform in Urban Middle Schools in Jilin Province}

The Academic Structure of PE Teachers in the Background of Sports Senior High School Entrance Examination Reform in Urban Middle Schools in Jilin Province. The development potential of the faculty is based on how many highly educated teachers. Although education is not directly related to the level of teaching, but the education and teachers' own knowledge literacy and knowledge theory there is a direct relationship, Sports senior high school entrance examination reform for student guidance and school development play an important role. With the country on the examination of the higher degree of emphasis on sports, the more rigorous examination in the examination, for teacher education, sports knowledge accumulation and technical action normative requirements are getting higher and higher.

Increase the competitiveness of the sports industry. All the time, school education there are heavy culture of light sports bad idea, while ignoring the physical exercise of students on the growth of psychological and physiological importance. The school in the recruitment of physical education teachers, there will be physical education teachers need to act as security, logistics and other backup work, do not value their academic level, as long as they can serve as "multi-functional" positions, therefore, lack of education on sports competition. All the time, school education has bad idea of heavy culture and light sports, while ignoring the physical exercise of students on the growth of psychological and physiological importance. The school in the recruitment of physical education teachers, there will be physical education teachers need to act as security, logistics and other backup work, do not value their academic level, as long as they can serve as "multi-functional" positions, As a result, sports employment lacks academic competition.

Sports senior high school entrance examination, in order to hold the rate of increase, the school for the physical education teachers of cultural knowledge and teaching literacy have great requirements, academic qualifications to recruit the quality of the conditions, which will exacerbate the competitiveness of sports teachers in sports Highly educated and high level of athletic ability to play the stage, you can eliminate dawdle and the lack of talent teachers, will play a variety of ability to improve the ability of sports and enhance the overall role of secondary sports. 
To promote the choice of physical education teachers to further study. Today, sports senior high school entrance examination has become the community, schools and parents have never been the focus of attention, to teach sports knowledge and skills, students in the limited exercise time to get better grades for the responsibility of physical education teachers, and have better education level.

In the context of the reform of sports in the examination, there are a large proportion of the physical education teachers who have undergraduate and college degrees, and some physical education teachers feel that the professional knowledge of education, teaching and training is not comprehensive in the course of physical education. For more up to the qualifications, to study junior college, graduate or master's degree higher education, to accept further and deeper research. With the physical education teachers tend to sports elite, to cultivate students in the test of sports training and targeted to improve the sports performance plays an extremely important role.

\section{The Structure of PE Teachers' Title in the Background of Sports senior high school entrance examination Reform in Urban Middle Schools in Jilin Province.}

The teacher title structure is the primary index to evaluate the level of school teaching and scientific research. The level of title is the performance of physical education teachers and experience, and its improvement will be of great significance to promote school sports subjects and transport sports talents.

Constraints on the Structure of the Title. The most proportion of PE Teachers on urban middle school of Jilin Province are intermediate title, which other titles are relatively small proportion. There is a positive correlation between the degree of emphasis on the subject and the title. When the school is under the prerequisite for further study, under the distribution of the title index, it will naturally consider the language, mathematics and English three subjects of the teacher, while ignoring some well-done physical education teachers, which let its face On this reality, they will think that the existence of difficulty and constraints, thus losing the enthusiasm of teaching, and even cause the loss of teachers.

Title is Linked to its Own Interests. In sports senior high school entrance examination, although the examination of sports scores only part of the total score, but play a school, parents and students pay great attention to the role of sports, the school organized a variety of sports activities, to carry out physical exercise longer. Physical education teachers rose to a status of attention, when the school title indicators, physical education teachers can account for a certain number, then they will realize that the title is positively related to income, the title of the level associated with their own interests, naturally increase Its enthusiasm and seriousness, improve the lack of places, effectively mobilize the overall development of teaching staff.

$\mathrm{C}$ the age structure of PE Teachers in the Background of Sports senior high school entrance examination Reform in Urban Middle Schools in Jilin Province

As the sports senior high school entrance examination through the test of a variety of projects to carry out physical considerations, physical education teachers need to make students skill skills to teach and accurate action demonstration, but this is the need to have a wealth of knowledge and experience, the teacher's age structure is reasonable As a teacher team forward momentum main premise.

The Present Situation of the Age Structure of Middle School PE Teachers in Jilin Province. Physical education teaching is not long-term attention, the school in the physical education teachers to pay attention to the degree is not high, physical education difficulties, so that the number of physical education school enrollment is small, the slow development of sports, physical education teachers appear as other subjects, leading to teaching experience There are many shortcomings in the shortage of old teachers, the level and quality of physical education and the lack of passion for physical education. With the reform of sports in the examination, sports status has been paid attention to, the demand for physical education teachers in schools has increased, and the proportion of PE students and middle school teachers has a large number of teaching experiences and methods, which contains a certain amount of knowledge savings, from maintaining the quality of physical education. At present, the proportion of young teachers in urban middle schools in Jilin Province is 
higher than that in the teaching, and the level of sports technology is high. The teaching work is highly complex and challenging. Middle-aged teachers occupy medium proportion, have certain teaching ability, Ability is slightly lacking; older teachers are basically less, but their teaching level is high.

\section{Reasonable Teacher's Age Structure}

With the reform of sports senior high school entrance examination, the importance of sports has been greatly increased, the demand for physical education teachers has increased, and the demand of physical education schools has also increased. Reasonable teacher's age structure should be the combination of old youth, old teachers have a wealth of teaching experience and scientific research ability, physical strength is slightly inadequate, can play a role in teaching guidelines, and young teachers on the contrary, the two can balance teaching level. Middle-aged teachers as the main faculty, which not only has long-term teaching experience, and has a certain energy and vitality. Effectively put the three together, in the rapid development of sports reform in the test, to maintain the teaching level.

The Gender differences of PE Teachers in the Background of Sports senior high school entrance examination Reform in Urban Middle Schools in Jilin Province. The proportion of female physical education teachers in urban middle schools in Jilin Province is less than that of male physical education teachers, which mainly restricts the professional play of female physical education teachers in family and society, weakening the personality and potential of women in sports industry.

Female Sports Teachers Generally Less Reasons. The choice of sports professional girls less physical education institutions to recruit female students are too few, resulting in female physical education teachers far less than male physical education teachers. Both from the sports technology or ability, the female teacher recognition is far lower than the male teachers, resulting in the emergence of female physical education teachers in the way of sports to find other industries. The school in the recruitment of physical education teachers when there is a bias on the female teachers that there are various problems, it is difficult to focus on the main spirit of the spirit of teaching, do not want to recruit female sports teachers. By the outdoor environmental conditions, resulting in the loss of female physical education teachers. Female physical education teachers exist body fat and physical decline fast, engaged in the lack of self-confidence in the sports industry and clear forward goals, limiting the development of women in sports professional.

The Advantages and Development of Female PE Teachers' Teaching. In the sports work, the female teachers have not only the potential of the sports industry, but also the female teachers can consider the physiological and psychological problems of the female students from the female point of view, and develop the scientific and reasonable exercise intensity and load to the middle school students. It can be fully developed in the sports program and the realization of physical education teaching objectives. However, in some sports to show beauty skills and highlight the rhythm of the sport, female teachers in the teaching will get better results, and in the examination of female students in the physical education is more likely to accept the guidance of female teachers, physical contact more convenient.

\section{The Team Teaching and Research Capacity Situation of PE Teachers in the Background of Sports Senior High School Entrance Examination Reform in Urban Middle Schools in Jilin Province}

Research situation of PE Teachers in the Background of Sports senior high school entrance examination Reform in Urban Middle Schools in Jilin Province. Now, to measure the overall quality of teachers is no longer simply through teaching methods, more importantly, can teach and research combined, through teaching and research, teachers can use their own life or experience to discover new laws in sports, new social value of the results, Deepen the research process, so as to obtain innovation, enhance teachers' own teaching quality and professional and technical skills.

The Situation of Scientific Research Papers Published by Urban Middle School PE 


\section{Teachers in Jilin Province 1.}

Table 1 Jilin Province urban middle school physical education teachers published nearly 10 years of sports research papers statistics table $(\mathrm{N}=885)$

\begin{tabular}{lcrrr}
\hline year & $2007-2009$ & $2010-2012$ & $2013-2015$ & $2016-2017$ \\
\hline Number of articles & 135 & 190 & 235 & 325 \\
percentage $(\%)$ & 15.2 & 21.5 & 26.6 & 36.7 \\
\hline
\end{tabular}

With the reform of sports senior high school entrance examination, the author puts forward the updated standards and goals for the teachers' teaching and research activities. The physical education teachers in middle schools in Jilin province began to attach importance to the teaching of physical education and scientific research, and promote the teaching and research. It is obvious from Table 1 that Jilin Province urban middle school physical education teachers published sports research papers accounted for $15.2 \%$ in 2007 to $2009,21.5 \%$ in 2010 to $2012,26.6 \%$ in 2013 to $2015,36.7 \%$ in 2016 to 2017, The number of published papers increased every year, physical education teachers more and more scientific awareness, reflecting the city of Jilin Province secondary school sports research work.

An Analysis of the Upward Trend of Scientific Research Papers Published by PE Teachers in Middle Schools in Jilin Province. Jilin Province urban middle school physical education teachers published nearly 10 years of sports research papers situation is on the rise, there are many factors contributed to this trend motive, mainly by the school, the physical education teachers and sports research to make an analysis.

(1)School side.

Jilin Province, the city middle school on the physical education teachers to support the high degree of scientific research to relax the funding of scientific research facilities, training, physical education teachers to raise the level of research and give certain incentives and research funding to encourage physical education teachers to spend time to do research, and carry out A variety of sports research on the training and seminars, the formation of a good atmosphere of scientific research.

(2)Physical education teachers themselves.

There are four aspects in the paper that the PE teachers in the urban middle schools in Jilin Province have published papers: The hard conditions for promotion and salary increase; The conditions for doing professional examination; To promote the development of physical education in depth; Like the sports industry.

(3)Sports research.

Sports science research is to develop the direction of sports development, is to improve the theoretical level of sports science prerequisite, help to improve the scientific literacy of athletes and business level. With the school more and more attention to sports research, sports research itself can be related to human health, and sports is everywhere in life, more attention by the community, greatly expanded sports research material.

The Importance of Scientific Research Ability of PE Teachers. For a physical education teacher, with a lot of professional knowledge and technology, is the basic ability to carry out scientific research, at the same time, the need to timely update the knowledge, so as to improve self-learning ability. 
Teaching workload of PE Teachers in the Background of Sports senior high school entrance examination Reform in Urban Middle Schools in Jilin Province

Table 2 Jilin Province urban middle school physical education teachers weekly workload statistics $(\mathrm{N}=89)$

\begin{tabular}{ccccc}
\hline Workload (hours / week) & 5 & $6-10$ & $11-14$ & 18 or more \\
\hline $\mathrm{N}$ & 0 & 9 & 52 & 28 \\
$\%$ & 0 & 10.1 & 58.4 & 31.5 \\
\hline
\end{tabular}

The Working Hours of PE Teachers in Middle Schools in Jilin Province. It is clear from Table 2 that $58.4 \%$ of the PE teachers have a weekly workload of 11-14 hours / week, and 31.5\% are in more than 18 hours. They do not include physical education, sports activities, training teams, Reflecting the weekly workload of physical education teachers are too large. Senior high school entrance examination score is occupied 40 sports senior high school entrance examination scores, schools and parents are of considerable importance, PE teachers by the task, need to develop a series of long-term sports training for senior high school entrance examination, and make the training plan according to the students at different physical conditions, but also with the reform of sports and students' physical conditions of senior high school entrance examination changes to make effective countermeasures.

Excessive Teaching Workload Hinders the Development of Sports Industry. Teaching staff over the General Assembly so that the loss of teaching teachers passion, all day busy in the cumbersome teaching, there will be tired emotions, resulting in physical education teacher instability, lazy to cope with teaching, will greatly reduce the teaching level. Physical education teachers do not have sufficient time to think and improve the teaching process, there is no time to improve their teaching ability and professional skills, in the overall state of flat, and even a downward trend.

Training and Learning of PE Teachers in the Background of Sports Senior High School Entrance Examination Reform in Urban Middle Schools in Jilin Province

Table 3 Jilin Province urban middle school physical education teacher training learning statistics table $(\mathrm{N}=89)$

\begin{tabular}{ccccccc}
\hline $\begin{array}{c}\text { Training } \\
\text { Method }\end{array}$ & online training & $\begin{array}{c}\text { On-the-job } \\
\text { training }\end{array}$ & $\begin{array}{c}\text { Self-stu } \\
\text { dy }\end{array}$ & $\begin{array}{c}\text { Lecture } \\
\text { training }\end{array}$ & Salon seminar & Other \\
\hline $\mathrm{N}$ & 18 & 9 & 10 & 34 & 16 & 2 \\
$\%$ & 20.2 & 10.1 & 11.2 & 38.2 & 18.1 & 2.2 \\
\hline
\end{tabular}

Jilin Province Urban Middle School Physical Education Teachers Continue to Receive Training. It is obvious from Table 3 that there are 34 physical education teachers in the city middle school of Jilin Province, and $38.2 \%$ of the total number of sports teachers. The training is welcomed by more sports teachers. At the same time, there are 18 people who choose online training, Accounting for 20.2\%; select the salon seminar in the middle position accounted for $18.1 \%$; on-the-job training and self-learning a little less people choose, respectively, accounted for $10.1 \%$ and $11.2 \%$. According to the data analysis, middle school teachers believe that physical education teachers through the training of the way the effect of better, professional guidance and communication, this method is the school more common training methods.

Combined with the Relevant Subjects for Training. Physical education teachers should continue to enrich their theoretical level, based on the original knowledge to deepen the use of 
theory and technology, its penetration of teaching, improve teaching quality and effectiveness. Want to have a solid sports professional knowledge and skills, but also need to master the relevant theoretical knowledge, such as psychology, multimedia, innovative education and other subjects, a comprehensive dissection of the truth of sports.

\section{Ideological quality of PE Teachers in the Background of Sports senior high school entrance examination Reform in Urban Middle Schools in Jilin Province}

Under the reform of sports in the examination, ideological and moral education as the main component, to strengthen the ideological quality of students to infiltrate and improve the moral cultivation of students is the most basic requirements of the teaching process.

Working Attitude of PE Teachers in the Background of Sports Senior High School Entrance Examination Reform in Urban Middle Schools in Jilin Province. Attitude is the expression of people's views on people or things in their words and deeds. The attitude of PE teachers determines the quality of the progress of school physical education, affecting teachers' own behavior and efficiency.

The Importance of Attitude of PE Teachers. In the physical education, students directly face the object is the teacher, the teacher should be based on different students, individualized, teachers in the classroom attitude anytime, anywhere because of a word or an action, inadvertently affect the students' thoughts. If the teacher itself is not serious about the classroom, once the students feel the teacher's attitude, will allow students to produce the same attitude to face the classroom.

The Correct Attitude of PE Teachers. Physical education teachers must face the correct attitude of students, model and exemplary role to guide students. Teaching process to vivid image of the language, the standard action to teach students to guide students to correct learning direction. Second, teachers should love education, care for students, proactive, optimistic and enterprising, and strive to cultivate new socialist people, and has excellent ideological quality and psychological quality, to keep teachers and students get along, so as to improve the quality of teaching.

\section{The Professionalism of PE Teachers in the Background of Sports Senior High School Entrance Examination Reform in Urban Middle Schools in Jilin Province}

The professionalism is a moral requirement for the attitude of society. The professionalism of physical education teachers is mainly to strengthen the sense of professionalism and responsibility to strengthen their professionalism.

Factors Affecting the Professional Spirit of PE Teachers

The School is not Enough to Support Sports Work. The school has always believed that physical education is dispensable, the physical education curriculum does not require students, usually school leaders and classroom surprise checks are not much, the school leaders do not value, resulting in the attitude of physical education teachers do not seriously, so students do not pay attention to physical education, sports The classroom lost the original meaning, serious physical education teachers to reduce the professionalism of the work.

Social Environment. The social environment directly affects the thought, emotion and behavior of PE teachers and whether they can give full play to their talents and values in teaching work. Society has always been the phenomenon of intellectual light, social status is low, social image is poor, and has been regarded as a deputy, in the marginal disciplines, which greatly affected the enthusiasm of physical education teachers, so that the physical education teacher is lost appeal.

PE Teachers Themselves. PE Teachers are not satisfied with the conditions of the post, coupled with the social and school management philosophy stagnation in the traditional wisdom of the concept of light and the pressure of the run, increased the physical education teachers in the negative emotions, making it easy to feel at ease Work, irresponsible to the work, lazy, dawdle, revival and other undesirable phenomena.

Strengthening the Cultivation of Physical Education Teachers' Professional Spirit

In accordance with the law of physical education teaching, adhere to the strengthening of 
physical fitness, to promote the coordinated development of students, so that students better completion of learning tasks, so as to achieve school education goals. In the process of training students to be fair and impartial, not favor the good results of students, but with the same enthusiasm and patience to teach students to make their healthy growth. In the teaching process need to patiently teach students knowledge, but also strict requirements of students to overcome the problems encountered in sports.

\section{Teaching Energy Input Situation of PE Teachers in the Background of Sports Senior High School Entrance Examination Reform in Urban Middle Schools in Jilin Province.}

Table 4 Jilin Province urban middle school physical education teachers teaching situation statistics $(\mathrm{N}=885)$

\begin{tabular}{ccccccc}
\hline project & $\begin{array}{c}\text { Training } \\
\text { team }\end{array}$ & PEclass & $\begin{array}{c}\text { Campus } \\
\text { Activities }\end{array}$ & $\begin{array}{c}\text { In the examination of } \\
\text { sports training }\end{array}$ & Part-time job & other \\
\hline$\%$ & 16.2 & 25.6 & 6.2 & 38.5 & 11.4 & 2.1 \\
\hline
\end{tabular}

It is obvious from Table 4 that the secondary school physical education teachers in Jilin Province believe that the teaching energy is mainly put into the middle school sports training and physical education, which accounted for $38.5 \%$ and $25.6 \%$ respectively. Teachers have a significant social responsibility, the current sports scores in Jilin Province accounted for 40 points in the test results, some other provinces accounted for 50 points, indicating that the score in Jilin Province, the possibility of rising sports scores, physical education is also subject to the school curriculum One, so the physical education teachers should have a sense of responsibility and dedication. Physical education teachers engaged in part-time accounted for $11.4 \%$, second only to the training team $16.2 \%$, although personal income increased, but will be scattered, affecting the development of sports industry. Jilin Province urban middle school physical education teachers believe that teaching energy is mainly devoted to campus activities accounted for $6.2 \%$, in the campus activities organization content, physical education teachers should focus on some students must meet the project, in addition, also pay attention to appropriate control and adjustment of campus activities Total and rhythm.

\section{Factors Affecting the Development of PE Teachers in the Background of Sports Senior High School Entrance Examination Reform in Urban Middle Schools in Jilin Province}

Lack of Teaching and Scientific Research. Jilin city middle school sports equipment aging and inadequate, the school funding is limited, can not buy a large number of equipment, carry out a wide range of courses and activities, the development of the sports industry is slow, scientific research difficult results.

Limited research conditions

Physical education teachers have limited scientific research conditions, can not play their own level in the field, resulting in weakened scientific research awareness, subjective mobility decline, scientific research once difficult to become difficult to continue, or even give up the idea.

The Number of PE Teachers and the Quality is not high. Most schools are generally a sports teacher with multiple classes of sports, and even some are responsible for some logistics work, the demand for physical education teachers greatly improved. Once the number of physical education teachers, the workload is large, naturally there is no sufficient energy and time to prepare lessons or do research, resulting in low quality teaching class.

Lack of Sports Equipment. Secondary school to buy sports equipment funds are limited, less active equipment or aging, low utilization rate is normal, and the venue is small, will limit the implementation of physical education.

The Change of PE Teachers' Educational Concept. Under the background of the reform of 
sports in the examination, it is necessary to make great changes in the realization of the new training plan, the choice of the training content and the improvement of the organization, the improvement of the method and the teaching evaluation. This requires physical education to break the old ideological bondage, in the teaching process to explore new strategies to accelerate the pace of development of sports teams.

\section{References}

[1] S.Y Zeng, Construction and development of higher education teachers in China [M]. Beijing: Aviation Industry Association, 1996:25.

[2] J.B Liu, J.G Ding, The status quo and development countermeasures of young and middle school teachers in our hospital [J]. Journal of Capital University of Physical Education and Sports, 2002, 12 (4): 26-27.

[3] X.P Deng, Deng Xiaoping's anthology [M]. Volume II, People's Publishing House, 1994: 108.

[4] An Ruliang. Prediction of the demand for senior professionals in physical education at the beginning of 21 [J]. Journal of Xi'an Physical Education University, 1999, 16 (3): 1-4.

[5] H.F Zhang, Attention should be paid to the humanities of teachers in physical education institutions [J]. Journal of Shandong Sport University, 2001, 17 (49): 72.

[6] D.M Liu, Experiences and lessons of teacher training in developed countries [J]. Journal of Henan Normal University, 2004, 14 (2): 5-7. 